\title{
The Characterization of Linear Polyethylene SRM's 1482, 1483, and 1484. II. Number-Average Molecular Weights by Membrane Osmometry
}

\author{
Herman L. Wagner and Peter H. Verdier \\ Institute for Materials Research, National Bureau of Standards, Washington, D.C. 20234
}

(October 19, 1977)

\begin{abstract}
Linear polyethylene Standard Reference Materials SRM 1482, 1483, and 1484 are certified for numberaverage molecular weight $M_{n}$. In this paper the experimental procedures employed for the determination of $M_{n}$ for these materials by membrane osmometry are described, and the techniques used to analyze the data and to estimate limits of systematic error are discussed.
\end{abstract}

Key words: Fraction; limiting viscosity number; membrane osmometry; narrow molecular weight distribution; number-average molecular weight; polyethylene; Standard Reference Material; weight-average molecular weight.

\section{Introduction}

Standard Reference Materials 1482, 1483, and 1484 are linear polyethylenes with relatively narrow distributions in molecular weight, issued by the National Bureau of Standards. Their general characteristics are described in the first paper of this series [1]. ${ }^{1}$ In the present paper, we describe the determination of their certificate values of numberaverage molecular weight by membrane osmometry. This well-established $[2,3]$ technique consists of measuring the equilibrium hydrostatic pressure difference between a solution and pure solvent separated by a membrane permeable to solvent alone, as a function of solution concentration.

\section{Experimental}

Osmotic pressure measurements were made with a Model 502 Hewlett-Packard Mechrolab Membrane Osmometer. ${ }^{2}$ This instrument, which has been described elsewhere [3], adjusts the hydrostatic pressure on the solvent side of the semipermeable membrane to achieve zero net liquid transfer across the membrane. The pressure is adjusted by varying the solvent level, which is measured with a resolution of 0.01 $\mathrm{cm}$, corresponding to a pressure difference of about $1 \mathrm{~Pa}$ for water or typical organic solvents. Gel cellophane membranes, type 450D, obtained from ArRo Labortories, Inc., were employed. Before use, they were conditioned to 1-chloronaphthalene, the solvent in which measurements were made, by a successive solvent-exchange procedure given in detail previously [4].

Solution temperatures at the membrane surface, which were in the range $125-130{ }^{\circ} \mathrm{C}$, could not be measured directly without risk of damage to the membrane. Temperatures were therefore monitored during osmotic pressure

1 Figures in brackets indicate literature references at the end of this paper.

2 Certain commercial equipment, instruments, or materials are identified in this paper in order to specify the experimental procedure. In no case does such identification imply recommendation or endorsement by the National Bureau of Standards, nor does it imply that the material or equipment identified is necessarily the best available for the purpose. measurements by a copper-constantan thermocouple springloaded against the stainless steel membrane clamps. In separate experiments, the temperature difference between this thermocouple and the membrane surface was determined by inserting a second thermocouple at the membrane surface.

Osmotic pressure differences were measured for solutions whose concentrations ranged from 0.7 to $3 \mathrm{~g} / \mathrm{L}$ for SRM 1482, 1 to $10 \mathrm{~g} / \mathrm{L}$ for SRM 1483, and 0.5 to $1.4 \mathrm{~g} / \mathrm{L}$ for SRM 1484. All solutions were made up directly by weight, without employing successive dilution techniques. Concentrations were calculated using values of solvent density and partial specific volume determined pycnometrically in this laboratory. The 1-chloronaphthalene was obtained from commercial material by distillation at reduced pressure after removal of residual naphthalene by sublimation, also at reduced pressure. Solutions were made up without adding antioxidant, since preliminary experiments suggested that its use led to erratic results, possibly due to the formation of gaseous decomposition products in the osmometer. No evidence of degradation was found in the course of this work. Reference readings, with solvent on both sides of the membrane, were taken before and after each solution reading, to take account of slow drifts due to changes in ambient pressure, etc.

\section{Results}

Number-average molecular weight $M_{n}$ may be obtained from the variation of osmotic pressure $\pi$ with solution concentration by means of the familiar virial expansion, expressed in one of the two equivalent forms:

$$
\begin{aligned}
\pi & =R T\left(M_{n}^{-1} c+A_{2} c^{2}+A_{3} c^{3}+\cdots\right) \\
& =\left(R T / M_{n}\right)\left(c+\Gamma_{2} c^{2}+\Gamma_{3} c^{3}+\cdots\right),
\end{aligned}
$$

where $c$ is solution concentration (weight per unit volume), $R$ and $T$ are the gas constant and the absolute temperature, respectively, and the $A$ 's and $\Gamma^{\prime}$ 's are the usual virial coefficients. In practice, the quantity actually measured is 
the difference $h$ in liquid level between solvent and solution, related to the osmotic pressure by $\pi=\rho g h$, where $\rho$ is solvent density and $g$ is the acceleration due to gravity. Thus, $M_{n}$ may be determined from the coefficient of the first power of concentration in a fit of osmotic pressure (or $h$ ) to a polynomial in solution concentration with no constant term. Since the concentrations chosen and the number of terms in eq (1) employed differ for the three Standard Reference Materials, we discuss them separately in the remainder of this section.

\subsection{SRM 1483}

Five subsets of measurements were made on SRM 1483. Each subset consisted of measurements on five solutions, with concentrations of approximately $1,2,3.5,5.5$, and 10 $\mathrm{g} / \mathrm{L}$, at a temperature of $128.6{ }^{\circ} \mathrm{C}$. The measured values of $h$, which ranged from 1 to $15 \mathrm{~cm}$, were fitted by unweighted least squares to a polynomial in the first, second, and third powers of solution concentration, and eq (1) was used to calculate $M_{n}, A_{2}$, and $A_{3}$ from the coefficients so obtained. The standard deviation in $h$ obtained from the least-squares analysis was $0.055 \mathrm{~cm}$, only slightly larger than the repeatability of the measurements. The values of $M_{n}$ and $A_{2}$ obtained are shown in table 1 , together with their standard deviations inferred from the least-squares fit. The value of $A_{2}$ is consistent with literature values for linear polyethylenes in 1-chloronaphthalene [4-7], and is in reasonable agreement with the value of $A_{2}$ obtained for SRM 1483 by light scattering cited in paper III of this series [8]. The value found for $A_{3}$ is $0.011 \mathrm{~mol} \mathrm{~cm} 6 / \mathrm{g}^{3}$, with a standard deviation of $0.012 \mathrm{~mol} \mathrm{~cm} / \mathrm{g}^{3}$. Thus, over the range of concentrations employed and to the precision of our measurements, we were unable to obtain a value for the third virial coefficient statistically significantly different from zero. This is hardly surprising, since at the highest concentrations employed, the contribution to the right-hand side of eq (1) from the second and higher virial coefficients is only about 40 percent of the term linear in $c$, and the contribution to the height difference $h$ from the third virial term is less than $0.4 \mathrm{~cm}$. Curiously, however, the ratio $\Gamma_{3} / \Gamma_{2}{ }^{2}=A_{3} /\left(M_{n} A_{2}{ }^{2}\right)$ has the value 0.3 , remarkably close to the often-employed estimate of $1 / 4$ for this ratio $[2,9]$.

\subsection{SRM 1482}

Five subsets of measurements were made on SRM 1482. Each subset consisted of measurements on four solutions, with concentrations of approximately $0.7,1,2$, and $3 \mathrm{~g} / \mathrm{L}$, at a temperature of $126.3^{\circ} \mathrm{C}$, and observed height differences $h$ ranging from 2 to $9 \mathrm{~cm}$. Preliminary analyses indicated a molecular weight of about $10^{4} \mathrm{~g} / \mathrm{mol}$ and a second virial coefficient roughly the same as that found for SRM 1483.
Using these values and the approximate relation $\Gamma_{3} / \Gamma_{2}{ }^{2}=$ $1 / 4$ previously cited, we can readily show that at the highest concentration measured, the contribution of the third virial term amounts to a height difference of only $0.002 \mathrm{~cm}$, well below the measuring capability of our equipment. Accordingly, for the analysis of the data the terms in $A_{3}$ and $\Gamma_{3}$ on the right-hand sides of eq (1) were ignored, and the measured values of $h$ were fitted by unweighted least squares to a polynomial in the first and second powers of concentration. The standard deviation in $h$ obtained from the fitting was $0.046 \mathrm{~cm}$, an acceptable value. The resulting values of $M_{n}$ and $A_{2}$ are given in table 1 , together with their standard deviations inferred from the least-squares fit.

\subsection{SRM 1484}

The experimental design for measurements on SRM 1484 was substantially different from that used for SRM's 1482 and 1483. If we take a nominal value of $10^{5} \mathrm{~g} / \mathrm{mol}$ for $M_{n}$ and take the value of $A_{2}$ found for SRM 1483 as a rough estimate of $A_{2}$ for SRM 1484, then the approximate relation $A_{3} /\left(M_{n} A_{2}^{2}\right)=1 / 4$ gives us an estimate of $3 \times 10^{-2}$ mol $\mathrm{cm}^{6} / \mathrm{g}^{3}$ for $A_{3}$. Using these estimates, we find that at a concentration of $10 \mathrm{~g} / \mathrm{L}$, the maximum employed for SRM 1483, the expected contributions to the measured height difference from the three terms on the right-hand side of eq (1) are $2.9 \mathrm{~cm}, 3.2 \mathrm{~cm}$, and $0.9 \mathrm{~cm}$, in that order. Thus, the second term is actually larger than the first, and it seems more than likely that the fourth and higher terms will contribute significantly. Since the primary purpose of this work is the determination of $M_{n}$, we restrict ourselves to measurements at and below a concentration $c_{\max }$ of $1.4 \mathrm{~g} / \mathrm{L}$, rather than include additional terms in eq (1). At $c_{\max }$, the expected contributions to the measured height difference will be about $0.4 \mathrm{~cm}, 0.07 \mathrm{~cm}$, and $0.003 \mathrm{~cm}$. The third term is well below the $0.01 \mathrm{~cm}$ resolution of the osmometer, and since the size of the terms is decreasing rapidly as we go to successively higher-order terms in concentration, we can be reasonably certain that the higher-order terms may be safely neglected.

Given the maximum concentration $c_{\max }$ to be used and the functional form to be fitted (eq (1) with the last term omitted), the expected precision with which $M_{n}$ may be estimated by a series of measurements of height difference $h$ at various concentrations not exceeding $c_{\max }$ is a function of the concentrations chosen. It can be shown [10] that for the present case, maximum precision in an estimate of $M_{n}$ is achieved by taking about one-sixth the measurements at $c_{\max }$ and the other five-sixths at a concentration roughly one-third $c_{\max }$. At this lower concentration, the predicted height difference is only about $0.14 \mathrm{~cm}$, much lower than is usually measured, and considerable replication is needed to obtain

TABLE 1. Molecular parameters obtained by membrane osmometry on solutions of linear polyethylene Standard Reference Materials 1482, 1483, and 1484 in 1-chloronaphthalene

Standard deviations are obtained from least-squares analyses of the data as described in the text.

\begin{tabular}{c|c|c|c|c|c|c}
\hline \hline & $\begin{array}{c}\text { Measurement } \\
\text { temperature, } \\
\text { Sample }\end{array}$ & $\begin{array}{c}\text { Number-average molec- } \\
\text { ular weight, } M_{n}, \mathrm{~g} / \mathrm{mol}\end{array}$ & $\begin{array}{c}\text { Standard deviation in } \\
M_{n}, \mathrm{~g} / \mathrm{mol}\end{array}$ & $\begin{array}{c}\text { Second virial coeffi- } \\
\text { cient, } A_{2}, \mathrm{~mol} \mathrm{~cm}^{3} / \mathrm{g}^{2}\end{array}$ & $\begin{array}{c}\text { Standard deviation in } \\
A_{2}, \mathrm{~mol}_{\mathrm{cm}} / \mathrm{g}^{2}\end{array}$ & $\begin{array}{c}\text { Number of } \\
\text { degrees of } \\
\text { freedom }\end{array}$ \\
\hline SRM 1482 & 126.3 & $1.1397 \times 10^{4}$ & $0.0080 \times 10^{4}$ & $1.34 \times 10^{-3}$ & $0.22 \times 10^{-3}$ & 18 \\
SRM 1483 & 128.6 & $2.895 \times 10^{4}$ & $.045 \times 10^{4}$ & $1.12 \times 10^{-3}$ & $.17 \times 10^{-3}$ & 22 \\
SRM 1484 & 129.7 & $1.005 \times 10^{5}$ & $.037 \times 10^{5}$ & $1.49 \times 10^{-3}$ & $.32 \times 10^{-3}$ & 34 \\
\hline
\end{tabular}


satisfactory precision in the final estimate of $M_{n}$. Accordingly, 30 measurements were made at a concentration of about $0.5 \mathrm{~g} / \mathrm{L}$, and 6 measurements at a concentration of about $1.4 \mathrm{~g} / \mathrm{L}$.

The measured values of $h$ were fitted to a polynomial in the first and second powers of concentration. The standard deviation in $h$ obtained from the fitting was $0.018 \mathrm{~cm}$. The values of $M_{n}$ and $A_{2}$ obtained are given in table 1, together with their standard deviations inferred from the least-squares fit.

\section{Systematic Errors}

We now list the likeliest sources of systematic error in the estimates of number-average molecular weight described in the preceding section, and attempt to set upper limits on their magnitudes. Individual sources of error are discussed in the following subsections; the resulting error-limit estimates are summarized in table 2 , expressed as percent errors applied to $M_{n}$.

TABLE 2. Percent errors in $\boldsymbol{M}_{n}$ introduced by measured quantities and approximations

\begin{tabular}{|c|c|c|c|}
\hline \multirow{2}{*}{ Source of error } & \multicolumn{3}{|c|}{ Error in $M_{n}$, percent } \\
\hline & SRM 1482 & SRM 1483 & SRM 1484 \\
\hline 1. Measurement temperature & 0.3 & 0.1 & 0.1 \\
\hline $\begin{array}{l}\text { 2. Solvent density in hydrostatic } \\
\text { column }\end{array}$ & 0.27 & 0.27 & 0.27 \\
\hline $\begin{array}{l}\text { 3. Solvent density at membrane } \\
\text { temperature }\end{array}$ & 0.3 & 0.25 & 0.25 \\
\hline 4. Solute weights & 0.60 & 0.85 & 0.19 \\
\hline 5. Solvent height scale & 0.56 & 0.99 & 3.04 \\
\hline $\begin{array}{l}\text { 6. Change in volume of solvent } \\
\text { system with solvent height }\end{array}$ & -0.06 & -0.06 & -0.06 \\
\hline 7. Bubble compression & -0.34 & -0.34 & -0.34 \\
\hline $\begin{array}{l}\text { 8. Permeation of solute through } \\
\text { membrane }\end{array}$ & +0.5 & +0.2 & \\
\hline $\begin{array}{l}\text { 9. Change in solution density with } \\
\text { concentration }\end{array}$ & -0.06 & -0.16 & -0.56 \\
\hline 10. Cutoff of virial expansion & +0.03 & +0.10 & +0.25 \\
\hline 11. Sum of positive errors & +0.53 & +0.3 & +0.25 \\
\hline 12. Sum of negative errors & -0.46 & -0.56 & -0.96 \\
\hline $\begin{array}{l}\text { 13. Maximum magnitude of error } \\
\text { from the signed errors com- } \\
\text { bined }\end{array}$ & 0.53 & 0.56 & 0.96 \\
\hline $\begin{array}{l}\text { 14. Root-sum-square of the above } \\
\text { maximum magnitude of error } \\
\text { and the unsigned errors }\end{array}$ & 1.10 & 1.47 & 3.22 \\
\hline $\begin{array}{l}\text { 15. Expected limit of systematic er- } \\
\text { rors from all sources, includ- } \\
\text { ing sources not identified and } \\
\text { treated here }\end{array}$ & $2 \%$ & $2 \%$ & $4 \%$ \\
\hline
\end{tabular}

In practice, $M_{n}$ is calculated from the relation:

$$
M_{n}=R T /\left(\rho_{h} g P\right),
$$

where

$R$ is the gas constant;

$T$ is the absolute temperature of the solution;

$\rho_{h}$ is the solvent density in the liquid column whose height differences $h$ measure the osmotic pressure;

$g$ is the acceleration due to gravity; and
$P$ is the coefficient of concentration $c$ in a fit of the height differences $h$ to a polynomial in $c$ of the form:

$$
h=P c+Q c^{2}+R c^{3}+\cdots .
$$

Of the quantities other than $P$ on the right-hand side of eq (2), only $T$ and $\rho_{h}$ can be in error by amounts sufficient to affect the final value of $M_{n}$ noticeably. Errors from these sources are discussed in sections 4.1 and 4.2. Since $P$ is the limit, as $c$ approaches zero, of the ratio $h / c$, systematic errors in $c$ and in $h$ will give rise to errors in $P$ and therefore in $M_{n}$. Systematic errors from these sources are discussed in sections 4.3 and 4.4 . Errors may also be introduced by the retention of too few terms on the right-hand side of eq (3) for the concentration range employed; errors from this source are discussed in section 4.5. Finally, the error limits from all the foregoing sources are combined and summarized in section 4.6 .

\subsection{Errors in Measurement Temperature}

As described in section 2, measurement temperatures were monitored by a thermocouple in contact with the clamps which support the membrane. The thermocouple itself is calibrated to $0.1{ }^{\circ} \mathrm{C}$; for SRM's 1483 and 1484 the principal error in measurement temperatures is due to the uncertainty in the temperature difference of about $1{ }^{\circ} \mathrm{C}$ between the monitoring thermocouple and the surface of the membrane. We believe that this uncertainty does not exceed $0.5{ }^{\circ} \mathrm{C}$. The resulting relative error in $M_{n}$, at a measurement temperature of about $400 \mathrm{~K}$, is seen from eq (2) to be $0.5 / 400$, or 0.1 percent for SRM's 1483 and 1484. The temperature control system was not working properly when measurements were made on SRM 1482, and the temperature varied over several degrees. We believe that the uncertainty in the effective average temperature for all the data is no greater than $1^{\circ} \mathrm{C}$, which would result in a relative error in $M_{n}$ of $1 / 400$, or 0.3 percent.

\subsection{Errors in Solvent Density}

Errors in solvent density affect the value of $M_{n}$ in two ways. First, the density $\rho_{h}$ of solvent in the hydrostatic head which balances the osmotic pressure difference between solvent and solution enters directly into the calculation of $M_{n}$ as shown by eq (2). Second, since solutions were made up by weight, rather than by volume, the value of $\rho_{0}$ of the solvent density at the measurement temperature affects the calculated values of solution concentrations and thus affects the calculated value of $P$ in eqs (2) and (3). The effect of errors in $\rho_{0}$ and $M_{n}$ is discussed in the following section. We believe that our measured values of $\rho_{h}$ are accurate to 0.2 percent at a given temperature. However, the temperature of the liquid column is uncontrolled, and is essentially room temperature. During this work, the fluctuations in room temperature were such as to make the effective average temperature uncertain by about $1{ }^{\circ} \mathrm{C}$. Measurements of the variation of the density of 1-chloronaphthalene with temperature in the vicinity of room temperature give a value of about 0.07 percent per ${ }^{\circ} \mathrm{C}$. Thus, the uncertainty in the temperature of the liquid column adds another 0.07 percent to the uncertainty in $\rho_{h}$. The total expected error in $\rho_{h}$ is therefore 0.27 percent, which by eq (2) leads to a possible error of 0.27 percent in $M_{n}$, for all three SRM's. 


\subsection{Errors in Solution Concentration}

As stated in section 2, solutions were made up by weight, and concentrations $c$ were calculated from the relation:

$$
c=w \rho_{0} /\left[1-w\left(1-\rho_{0} \bar{v}\right)\right],
$$

where $w$ and $\bar{v}$ are the weight fraction and partial specific volume, respectively, of solute in the solution. Thus, errors in $c$ can arise from errors in $w, \rho_{0}$, and $\bar{v}$. However, the value of $P$, and therefore of $M_{n}$, is unaffected by errors in $\bar{v}$. To see this, we observe that $P$ is the limit, as $c$ approaches zero, of the ratio $h / c$. However, $c$ may also be written as the product of $w$ and the solution density $\rho$. As $c$ approaches zero, $\rho$ of course approaches $\rho_{0}$, and $P$ can be re-expressed as $\rho_{0}^{-1}$ times the zero-concentration limit of $h / w$, and is therefore independent of $\bar{v}$ and inversely proportional to $\rho_{0}$. The relative error in $M_{n}$ is therefore independent of the error in $\bar{v}$ and equal in magnitude to the relative error in $\rho_{0}$. We believe that the accuracy of our measurement of $\rho_{0}$ is 0.2 percent at a given temperature. However, as with $\rho_{h}$, the uncertainties in measurement temperature discussed in section 4.1 increase this figure by 0.1 percent for SRM 1482 and by 0.05 percent for SRM's 1483 and 1484. Therefore, our final estimates for errors in $M_{n}$ arising from errors in $\rho_{0}$ are: 0.3 percent for SRM 1482, 0.25 percent for SRM's 1483 and 1484 .

Solute and solution weights were measured on semimicro balances accurate to $0.1 \mathrm{mg}$. The balance used to measure solute weights for SRM 1484 was checked at the values of solute weight actually employed and was found to be accurate to 0.1 percent at those values. In order to estimate the effect of these weighing uncertainties on the values of $M_{n}$, a series of comparison calculations was carried out. For each SRM, a reference subset of typical data points was chosen, one at each concentration measured, and a "reference" value of $M_{n}$ was calculated from this set of points. The value of each solute weight in turn was then increased by its assumed limit of error, and the value of $M_{n}$ recalculated. The resulting percent changes in the reference values of $M_{n}$ are shown in table 3, together with the sum of the absolute values of the individual changes, their algebraic sum, and the square root of the sum of their squares (root-sum-square). The sum of the absolute values represents the error in the case where every weighing is in error by the maximum amount possible and in the direction which maximizes the resulting error in $M_{n}$. We reject this estimate as overly pessimistic. The algebraic sum would be the appropriate measure if all the weighings were in error by the same amount, and the root-sum-square would be appropriate if the individual errors were of random sign. Since both these possibilities seem physically plausible, we select as our error estimate the larger of the absolute value of the algebraic sum and the root-sum-square. This turns out to be the algebraic sum for SRM's 1482 and 1483, and the rootsum-square for SRM 1484; the corresponding values are shown in line 4 of table 2.

\subsection{Errors in Solvent Heights}

Errors in the scale used to measure the solvent heights $h$ will of course cause errors in $P$ and therefore in $M_{n}$. The scale was therefore spot-checked with a cathetometer over its entire range. The largest discrepancy found was $0.012 \mathrm{~cm}$. This value was therefore used to obtain error estimates for SRM's 1482 and 1483. However, for SRM 1484, with much smaller measured height differences than the other two, a more precise error limit was needed. The scale was therefore rechecked every $0.01 \mathrm{~cm}$ over the region in which height differences were measured for SRM 1484. Over this very limited region of the scale, the largest discrepancy found was $0.0031 \mathrm{~cm}$, and this value was used to obtain error estimates for SRM 1484. The errors in $M_{n}$ resulting from the assumed errors in $h$ were obtained by the same kind of comparison calculation described in section 4.3 for errors in solute weight; the results are shown in table 3 . Again, we reject the sum of the absolute values of the individual changes as being too pessimistic and choose the larger of the absolute value of the algebraic sum of the individual changes and their rootsum-square. As with the errors due to solute weights, this turns out to be the absolute value of the algebraic sum for SRM's 1482 and 1483, and the root-sum-square for SRM 1484; the corresponding values are given in line 5 of table 2 .

TABLE 3. Percent errors in number-average molecular weight $M_{n}$ introduced by assumed errors in solute weight and and in solvent heights

\begin{tabular}{|c|c|c|c|}
\hline & SRM 1482 & SRM 1483 & SRM 1484 \\
\hline Number of solutions in reference subset & 4 & 5 & 2 \\
\hline $\begin{array}{l}\text { Percent changes in } M_{n} \text { from assumed errors in } \\
\text { solute weight }\end{array}$ & $\begin{array}{l}0.23,-0.16 \\
0.29,0.24\end{array}$ & $\begin{array}{l}0.34,0.44 \\
0.37,-0.34 \\
0.04\end{array}$ & $\begin{array}{r}0.17 \\
-0.08\end{array}$ \\
\hline Algebraic sum of above & 0.60 & 0.85 & 0.09 \\
\hline Sum of absolute values of above & 0.92 & 1.53 & 0.25 \\
\hline Root-sum-square of above & 0.47 & 0.75 & 0.19 \\
\hline $\begin{array}{l}\text { Percent changes in } M_{n} \text { from assumed errors in } \\
\text { solvent height }\end{array}$ & $\begin{array}{l}-0.23,0.15 \\
-0.29,-0.19\end{array}$ & $\begin{array}{l}-0.40,-0.48 \\
-0.38,0.29 \\
-0.02\end{array}$ & $\begin{array}{r}-3.01 \\
0.46\end{array}$ \\
\hline Sum of absolute values of above & 0.86 & 1.57 & 3.47 \\
\hline Algebraic sum of above & -0.56 & -0.99 & -2.55 \\
\hline Root-sum-square of above & 0.44 & 0.79 & 3.04 \\
\hline
\end{tabular}


Errors in solvent height can also arise from changes in the volume of the solvent reservoir system with height. The quantity actually measured is not strictly the liquid level of solvent, but rather the position of a reservoir of solvent connected to the osmometer by flexible tubing. If the volume of the tubing changes slightly with changes in the height of the reservoir, the liquid level within the reservoir will change, and the true height difference between solvent and solution will be the difference in scale reading plus the change in solvent level within the reservoir at the two heights. This error was estimated by comparing the difference in solvent meniscus level at two positions near the top and bottom of the total range of $40 \mathrm{~cm}$, measured directly with a cathetometer, with the difference in osmometer scale readings at the two positions. The difference in the scale readings was found to be $0.022 \mathrm{~cm}$ larger than the difference measured with the cathetometer. This amounts to a scaling error in $h$ of $0.022 / 40$ or 0.06 percent, and a corresponding error in $M_{n}$ of -0.06 percent, shown in line 6 of table 2 .

Changes in the length of the control bubble whose motion is used to sense liquid flow give rise to a third source of error in solvent height. When the solvent level changes in response to changes in solution concentration, the hydrostatic pressure on the control bubble changes and the bubble expands or contracts. Treating the bubble as a perfect gas, which is adequate for our present purpose, we can easily show that the relative error in $h$ is given by $b \rho_{h} g / P_{0}$, where $b$ is the length of the bubble and $P_{0}$ is atmospheric pressure. Assuming a maximum bubble length of $3 \mathrm{~cm}$ (a very safe upper limit), we find an error in $h$ from this source of 0.34 percent. The control bubble is located directly underneath the membrane. Its expansion when the concentration of solution in the osmometer is increased therefore gives rise to an apparent increase in $h$, or a decrease in the apparent value of $\boldsymbol{M}_{n}$. The resulting error in $M_{n}$ of -0.34 percent is shown in line 7 of table 2.

Measured values of $h$ will be too low if the membrane is not completely impermeable to solute. Although experience suggests that permeation should be negligible for the membranes and solutes employed in this work, limits of error from this source were estimated for SRM's 1482 and 1483 as described below.

Membrane permeation effects were estimated for SRM 1482 by comparing the values of $h$ obtained for a solution of SRM 1482 using the 450D membranes normally employed in this work with the values obtained for the same solution with an appreciably thicker membrane (ArRo 600D). The measured value of $h$ was about $13 \mathrm{~cm}$, and the value obtained with the thicker membrane was larger than that obtained with the normal membrane by $0.03 \pm 0.04 \mathrm{~cm}$. Taking the worst case, we have a maximum difference of $0.07 \mathrm{~cm}$, which implies a maximum relative error in $M_{n}$ of $0.07 / 13$, or +0.5 percènt.

Errors from membrane permeation were estimated for SRM 1483 by looking for a slow decrease in $h$ with time resulting from such leakage. No such drifts were observed for a solution which gave a value of $h$ of about $13 \mathrm{~cm}$. We estimate that we could have observed a drift of $0.01 \mathrm{~cm}$, and that such a drift might imply a total decrease of $0.02 \mathrm{~cm}$, allowing for instrumental response time. We therefore estimate the limit of error from membrane permeation for SRM 1483 as $0.02 / 13$, or +0.2 percent.
Finally, the difference in density between solvent and solution gives rise to an error in $h$. In practice, $h$ is measured as the change in reading when solution in the sample side of the osmometer is replaced by solvent. The sample tube is filled to the same height in both cases. If the densities of solvent and solution are different, as is usually the case, replacing solution by solvent will give rise to a purely hydrostatic pressure change, and the observed value of $h$ will be the sum of this change and the change arising from the osmotic pressure difference across the membrane. The change in hydrostatic pressure is just $a g\left(\rho-\rho_{0}\right)$, where $a$ is the height above the membrane surface to which the sample tube is filled and $\rho$ is the solution density. The resulting change in the measured $h$ is then $a\left(\rho_{0}-\rho\right) / \rho_{h}$. The difference $\rho_{0}-\rho$ is given by $c\left(\rho_{0} \bar{v}-1\right)$, so the error in $h$ is just $\operatorname{ac}\left(\rho_{0} \bar{v}-1\right) / \rho_{h}$. Using eqs (2) and (3), we find that the resulting relative error in $M_{n}$ is given by:

$$
M_{n} \operatorname{ag}\left(1-\rho_{0} \bar{v}\right) /(R T) \text {. }
$$

For our osmometer and operating conditions, this error is -0.06 percent, -0.16 percent, and -0.55 percent for SRM's 1482, 1483, and 1484, respectively, as shown in line 9 of table 2 .

\subsection{Errors due to Cutoff of Virial Expansion}

As discussed in section 3, only the first two terms on the right-hand side of eq (1) were employed for the analysis of the data for SRM's 1482 and 1484. Although contributions from the third and higher virial coefficients are smaller than the resolution of the osmometer, their neglect nevertheless constitutes a source of systematic error. In order to estimate the magnitude of the error involved, we estimate the third term on the right-hand side of eq (1) by making the assumption $\Gamma_{3}=\Gamma_{2}^{2} / 4$. As discussed in section 3 , the data obtained for SRM 1483 are at least not inconsistent with this assumption. This allows us to estimate the contribution to $h$ of the third term on the right-hand side of eq (3). Its neglect in the analysis may be treated as an error in the measured $h$ at each concentration measured; the resulting error in $M_{n}$ may then be obtained by making use of the estimates of the effect of errors in $h$ on $M_{n}$ obtained by comparison calculations as described in section 4.4. In this case, since the "errors" in $h$ are of the same sign for all the measured solutions, we take the algebraic sum of the individual changes in $M_{n}$ given in table 3 , each scaled by the ratio of the third virial term in $h$ to the error in $h$ assumed in table 3 . The resulting errors of +0.03 percent and +0.25 percent for SRM's 1482, and 1484, respectively, are shown in line 10 of table 2.

For SRM 1483, contributions from the third virial coefficient are already included in the analysis. In this case, we wish to set bounds on the possible contributions from the fourth and higher virial coefficients. In the absence of any means of estimating the fourth virial coefficient theoretically, we resort to the expedient of examining the relative sizes of the three terms in the right-hand side of eq (1) at the highest concentration measured. They turn out to be in the ratio 1:0.324:0.026. Thus, the third virial term is only about 8 percent of the second, and the second is about 32 percent of the first. It seems adequately cautious to assume that as a 
maximum, the fourth term will be to the third as the second is to the first. Then we have as a maximum estimate:

$$
\Gamma_{4}=\Gamma_{2} \Gamma_{3}
$$

Treating the contribution to $h$ from a hypothetical fourth virial coefficient of this magnitude as an error in $h$, in a manner analogus to that described above for the third virial terms for SRM's 1482 and 1484, we obtain an estimated error in $M_{n}$ for SRM 1483 of +0.10 percent, as shown in line 10 to table 2 .

\subsection{Summary}

Estimates of the contributions of individual sources to the overall systematic error in $M_{n}$ are summarized in table 2 . Errors which may be in either direction are shown unsigned; errors which can only be in one direction are shown with the appropriate signs. We believe that for errors which can be either positive or negative, the sum of the absolute values of the individual contributions gives an overly pessimistic estimate of total error. In addition, the total error from all the signed sources together cannot exceed the greater of the sum of all the positive errors, shown in line 11 of table 2, and the negative of the sum of all the negative errors, shown in line 12. This quantity, the maximum possible error arising from the signed error sources, is shown in line 13. In order to combine its effects with those of the unsigned error estimates, we form the root-sum-square, shown in line 14. Finally, to take account of any sources of error not explicitly considered here, we round each of the estimates so obtained upward to the next whole percent. The resulting estimates of limits of systematic error, shown on line 15 of table 2, are those given on the certificates for SRM's 1482, 1483, and 1484 .

\section{References}

[1] Verdier, P. H., and Wagner, H. L., The Characterization of Linear Polyethylene SRM's 1482, 1483, and 1484. I. Introduction., J. Res. Nat. Bur. Stand. (U.S.), 83, No. 2, (Mar.-Apr. 1978).

[2] See, for example, Flory, P. J., Principles of Polymer Chemistry (Cornell University Press, 1953).

[3] Coll, H., and Stross, F. H., Determination of Molecular Weights by Equilibrium Osmotic-Pressure Measurements, in Characterization of Macromolecular Structure (Publication 1573, National Academy of Sciences, Washington, D. C., 1968).

[4] Brown, J. E., and Verdier, P. H., The Characterization of Linear Polyethylene SRM 1475. IX. Number Average Molecular Weight of Fractions by Membrane Osmometry, J. Res. Nat. Bur. Stand. (U.S.) 76A, No. 2, 161-163 (Mar.-Apr. 1972).

[5] Kokle, V., Billmeyer, F. W., Jr., Muus, L. T., and Newitt, E. J., The Molecular Structure of Polyethylene. XI. Weight- and NumberAverage Molecular Weights of Selected Samples, J. Polym. Sci. 62, 251-261 (1962).

[6] Chiang, R., Intrinsic Viscosity-Molecular Weight Relationship for Fractions of Linear Polyethylene, J. Phys. Chem. 69, 1645-1653 (1965).

[7] Frolen, L. J., Ross, G. S., Wims, A. M., and Verdier, P. H., The Characterization of Linear Polyethylene SRM 1475. VIII. LightScattering Studies on Polyethylenes in 1-Chloronaphthalene, J. Res. Nat. Bur. Stand. (U.S.) 76A, No. 2, 156-160 (Mar.-Apr. 1972).

[8] Han, C. C., Verdier, P. H., and Wagner, H. L., The Characterization of Linear Polyethylene SRM's 1482, 1483, and 1484. III. WeightAverage Molecular Weights by Light Scattering, J. Res. Nat. Bur. Stand. (U.S.) 83, No. 2 (Mar.-Apr. 1978).

[9] See, for example, Morawetz, H., Macromolecules in Solution (Interscience, 1965), p. 174.

[10] Verdier, P. H., to be published. 